\title{
CLASSIFICATION(S) OF DANIELEWSKI HYPERSURFACES
}

\author{
P.-M. POLONI
}

\begin{abstract}
The Danielewski hypersurfaces are the hypersurfaces $X_{Q, n}$ in $\mathbb{C}^{3}$ defined by an equation of the form $x^{n} y=Q(x, z)$ where $n \geq 1$ and $Q(x, z)$ is a polynomial such that $Q(0, z)$ is of degree at least two. They were studied by many authors during the last twenty years. In the present article, we give their classification as algebraic varieties. We also give their classification up to automorphism of the ambient space. As a corollary, we obtain that every Danielewski hypersurface $X_{Q, n}$ with $n \geq 2$ admits at least two non-equivalent embeddings into $\mathbb{C}^{3}$.
\end{abstract}

\section{INTRODUCTION}

The story of Danielewski hypersurfaces goes back to 1989, when Danielewski [4 showed that, if $W_{n}$ denotes the hypersurface in $\mathbb{C}^{3}$ defined by the equation $x^{n} y-z(z-1)=0$, then $W_{n} \times \mathbb{C}$ and $W_{m} \times \mathbb{C}$ are isomorphic algebraic varieties for all $n, m \geq 1$, whereas the surfaces $W_{1}$ and $W_{2}$ are not isomorphic. He discovered the first counterexamples to the Cancellation Problem. Then, Fieseler [6] proved that $W_{n}$ and $W_{m}$ are not isomorphic if $n \neq m$.

Since these results appeared, complex algebraic surfaces defined by equations of the form $x^{n} y-Q(x, z)=0$ (now called Danielewski hypersurfaces) have been studied by many different authors (see [10], 8], 3], 1], 7], 9]), leading to new interesting examples as byproducts. Let us mention two of them.

In their work on embeddings of Danielewski hypersurfaces given by $x^{n} y=p(z)$, Freudenburg and Moser-Jauslin [7] discovered an example of two smooth algebraic surfaces which are algebraically non-isomorphic but holomorphically isomorphic.

More recently, the study of Danielewski hypersurfaces of equations $x^{2} y-z^{2}-x q(z)=0$ produced the first counterexamples to the Stable Equivalence Problem [9]; that is two polynomials of $\mathbb{C}\left[X_{1}, X_{2}, X_{3}\right]$ which are not equivalent (i.e. such that there exist no algebraic automorphism of $\mathbb{C}\left[X_{1}, X_{2}, X_{3}\right]$ which maps one to the other one) but, when considered as polynomials of $\mathbb{C}\left[X_{1}, X_{2}, X_{3}, X_{4}\right]$, become equivalent.

The purpose of the present paper is to classify all Danielewski hypersurfaces, both as algebraic varieties, and also as hypersurfaces in $\mathbb{C}^{3}$. More precisely, we will give necessary and sufficient conditions for isomorphism of two Danielewski hypersurfaces; and, on the other hand, we will give necessary and sufficient conditions for equivalence of two isomorphic Danielewski hypersurfaces. Recall that two isomorphic hypersurfaces $H_{1}, H_{2} \subset \mathbb{C}^{n}$ are said to be equivalent if there exists an algebraic automorphism $\Phi$ of $\mathbb{C}^{n}$ which maps one to the other one, i.e. such that $\Phi\left(H_{1}\right)=H_{2}$.

We know indeed that isomorphic classes and equivalence classes are distinct for Danielewski hypersurfaces. This was first observed by Freudenburg and Moser-Jauslin, who showed in [7] that the Danielewski hypersurfaces defined respectively by the equations $f=x^{2} y-(1+x)\left(z^{2}-\right.$ 1) $=0$ and $g=x^{2} y-z^{2}+1=0$ are isomorphic but non equivalent. (One way to see that they are not equivalent is to remark that the level surfaces $f^{-1}(c)$ are smooth for every constant $c \in \mathbb{C}$, whereas the surface $g^{-1}(1)$ is singular along the line $\{x=z=0\}$.)

Several papers already contain the classification, up to isomorphism, of Danielewski hypersurfaces of a certain form. Makar-Limanov proved in [8] that two Danielewski hypersurfaces of equations $x^{n_{1}} y-p_{1}(z)=0$ and $x^{n_{2}} y-p_{2}(z)=0$ with $n_{1}, n_{2} \geq 2$ and $p_{1}, p_{2} \in \mathbb{C}[z]$ are isomorphic if and only if they are equivalent via an affine automorphism of the form $(x, y, z) \mapsto(a x, b y, c z+d)$ with $a, b, c \in \mathbb{C}^{*}$ and $d \in \mathbb{C}$. Then, Daigle generalized in [3] this 
result to the case $n_{1}, n_{2} \geq 1$. Next, Wilkens has given in [10] the classification of Danielewski hypersurfaces of equations $x^{n} y-z^{2}-h(x) z=0$ with $n \geq 2$ and $h(x) \in \mathbb{C}[x]$.

Finally, Dubouloz and the author showed in [5] that every Danielewski hypersurface $X_{Q, n}$ of equation $x^{n} y=Q(x, z)$, where $Q(x, z)$ is such that $Q(0, z)$ has simple roots, is isomorphic to a one defined by an equation of the form $x^{n} y=\prod_{i=1}^{d}\left(z-\sigma_{i}(x)\right)$, where $\left\{\sigma_{1}(x), \ldots \sigma_{d}(x)\right\}$ is a collection of polynomials in $\mathbb{C}[x]$ so that $\sigma_{i}(0) \neq \sigma_{j}(0)$ if $i \neq j$. In the same paper, we classified these last ones and called them standard forms. This effectively classifies, up to isomorphism, all Danielewski hypersurfaces of equations $x^{n} y=Q(x, z)$, where $Q(0, z)$ has simple roots.

In the present paper, we generalize the notion of Danielewski hypersurface in standard form and we prove that every Danielewski hypersurface is isomorphic to a one in standard form (which can be found by an algorithmic procedure). Then, we are able to classify all Danielewski hypersurfaces. The terminology standard form is relevant since every isomorphism between two Danielewski hypersurfaces in standard form - and every automorphism of such a Danielewski hypersurface - extend to a triangular automorphism of $\mathbb{C}^{3}$.

We also give a criterion (Theorem 4.1) to distinguish isomorphic but not equivalent Danielewski hypersurfaces.

As a corollary, we obtain that every Danielewski hypersurface defined by an equation of the form $x^{n} y-Q(x, z)=0$ with $n \geq 2$ admits at least two non equivalent embeddings into $\mathbb{C}^{3}$.

Most of these results are based on a precise picture of the sets of locally nilpotent derivations of coordinate rings of Danielewski hypersurfaces, obtained using techniques which were mainly developed by Makar-Limanov in [8].

The paper is organized as follows. Section 1 is the introduction. In section 2, we fix some notations and definitions. In section 3, we study the locally nilpotent derivations on the Danielewski hypersurfaces in order to get information on what an isomorphism between two Danielewski hypersurfaces looks like. Section 4 is devoted to the classification of Danielewski hypersurfaces up to equivalence, whereas sections 5 and 6 contain their classification up to isomorphism and the study of the Danielewski hypersurfaces in standard form.

\section{Definitions And notations}

In this paper, our base field is $\mathbb{C}$, the field of complex numbers. If $n \geq 1$, then $\mathbb{C}^{[n]}$ will denote a polynomial ring in $n$ variables over $\mathbb{C}$.

Definition 2.1. Two hypersurfaces $X_{1}$ and $X_{2}$ of $\mathbb{C}^{n}$ are said to be equivalent if there exists a (polynomial) automorphism $\Phi$ of $\mathbb{C}^{n}$ such that $\Phi\left(X_{1}\right)=X_{2}$.

This notion is related to the notion of equivalent embeddings in the following sense. If $X_{1}$ and $X_{2}$ are two isomorphic hypersurfaces of $\mathbb{C}^{n}$ which are not equivalent, then $X_{1}$ admits two non-equivalent embeddings into $\mathbb{C}^{n}$. More precisely, let $\varphi: X_{1} \rightarrow X_{2}$ be an isomorphism and denote $i_{1}: X_{1} \rightarrow \mathbb{C}^{n}$ and $i_{2}: X_{2} \rightarrow \mathbb{C}^{n}$ the inclusion maps. Then, $i_{1}$ and $i_{2} \circ \varphi$ are two non-equivalent embeddings of $X_{1}$ into $\mathbb{C}^{n}$, since $\varphi$ does not extend to an automorphism of $\mathbb{C}^{n}$.

Definition 2.2. A Danielewski hypersurface is a hypersurface $X_{Q, n} \subset \mathbb{C}^{3}$ defined by an equation of the form $x^{n} y-Q(x, z)=0$, where $n \in \mathbb{N}$ and $Q(x, z) \in \mathbb{C}[x, z]$ is such that $\operatorname{deg}(Q(0, z)) \geq 2$.

We will denote by $S_{Q, n}$ the coordinate ring of a Danielewski hypersurface $X_{Q, n}$, i.e. $S_{Q, n}=$ $\mathbb{C}\left[X_{Q, n}\right]=\mathbb{C}[x, y, z] /\left(x^{n} y-Q(x, z)\right)$.

It can be easily seen that every Danielewski hypersurface is equivalent to a one of the form $X_{Q, n}$ with $\operatorname{deg}_{x} Q(x, z)<n$.

Lemma 2.3. Let $X_{Q, n}$ be a Danielewski hypersurface and $R(x, z) \in \mathbb{C}[x, z]$ be a polynomial. Then $X_{Q, n}$ is equivalent to the Danielewski hypersurface of equation $x^{n} y-Q(x, z)-x^{n} R(x, z)$.

Proof. It suffices to consider the triangular automorphism of $\mathbb{C}^{3}$ defined by $(x, y, z) \mapsto(x, y+$ $R(x, z), z)$. 


\section{USING LOCALLY NILPOTENT DERIVATIONS}

One important property of Danielewski hypersurfaces is that they admit nontrivial actions of the additive group $\mathbb{C}_{+}$. For instance, we can define a $\mathbb{C}_{+}$-action $\delta_{Q, n}: \mathbb{C} \times X_{Q, n} \rightarrow X_{Q, n}$ on a hypersurface $X_{Q, n}$ by posing

$$
\delta_{Q, n}(t,(x, y, z))=\left(x, y+x^{-n}\left(Q\left(x, z+t x^{n}\right)-Q(x, z)\right), z+t x^{n}\right) .
$$

Since a $\mathbb{C}_{+}$-actions on an affine complex surface $S$ induces a $\mathbb{C}$-fibration over an affine curve, affine complex surfaces with $\mathbb{C}_{+}$-actions split into two cases. Either there is only one $\mathbb{C}$-fibration on $S$ up to an isomorphism of the base, or there exists a second one. In other words, either the surface has a Makar-Limanov invariant of transcendence degree one, or its Makar-Limanov invariant is trivial. Recall that algebraic $\mathbb{C}_{+}$-actions on an affine variety $\operatorname{Spec} A$ correspond to locally nilpotent derivations on the $\mathbb{C}$-algebra $A$ (for example, the action $\delta_{Q, n}$ on a surface $X_{Q, n}$ corresponds to the locally nilpotent derivation $\Delta_{Q, n}=x^{n} \frac{\partial}{\partial z}+\frac{\partial Q(x, z)}{\partial z} \frac{\partial}{\partial y}$ on the coordinate ring $\left.\mathbb{C}\left[X_{Q, n}\right]\right)$, and that the Makar-Limanov invariant $\operatorname{ML}(A)$ of an algebra $A$ is defined as the intersection of all kernels of locally nilpotent derivations of $A$. Equivalently, $\operatorname{ML}(A)$ is the intersection of all invariant rings of algebraic $\mathbb{C}_{+}$-actions on $\operatorname{Spec}(A)$.

Applying Makar-Limanov's techniques, one can obtain the first important result concerning Danielewski hypersurfaces: the Makar-Limanov invariant of a Danielewski hypersurface $X_{Q, n}$ is non-trivial if $n \geq 2$.

Theorem 3.1. Let $X_{Q, n}$ be a Danielewski hypersurface. Then $\operatorname{ML}\left(X_{Q, n}\right)=\mathbb{C}$ if $n=1$ and $\operatorname{ML}\left(X_{Q, n}\right)=\mathbb{C}[x]$ if $n \geq 2$.

Proof. Let $X_{Q, n}$ be a Danielewski hypersurface.

If $n=1$, the result is easy. Indeed, we can suppose that $Q(x, z)=p(z) \in \mathbb{C}[z]$ (see Lemma 2.3). Then, it suffices to consider the following locally nilpotent derivations on the coordinate ring $S_{p, 1}=\mathbb{C}[x, y, z] /(x y-p(z))$.

$$
\delta_{1}=x \frac{\partial}{\partial z}+p^{\prime}(z) \frac{\partial}{\partial y} \quad \text { and } \quad \delta_{2}=y \frac{\partial}{\partial z}+p^{\prime}(z) \frac{\partial}{\partial x} .
$$

Since $\operatorname{Ker}\left(\delta_{1}\right) \cap \operatorname{Ker}\left(\delta_{2}\right)=\mathbb{C}$, this shows that the Makar-Limanov invariant of every Danielewski hypersurface $X_{Q, 1}$ is trivial.

Suppose now that $n \geq 2$ and let $\delta$ be a non-zero locally nilpotent derivation on the coordinate $\operatorname{ring} S_{Q, n}=\mathbb{C}\left[X_{Q, n}\right]$. Without loss of generality, we can suppose that the leading term of $Q(0, z)$ is $z^{d}$ with $d \geq 2$.

Then, the proof given by Makar-Limanov in [8] for hypersurfaces of equation $x^{n} y=p(z)$ still holds. This proof goes as follows.

The main idea is to consider $S_{Q, n}$ as a subalgebra of $\mathbb{C}\left[x, x^{-1}, z\right]$ with $y=x^{-n} Q(x, z)$ and to choose a $\mathbb{Z}$-filtration on $S_{Q, n}$ such that the corresponding graded algebra $\operatorname{Gr}\left(S_{Q, n}\right)$ is isomorphic to the subalgebra $\mathbb{C}\left[x, x^{-n} z^{d}, z\right]$.

Recall that Makar-Limanov has proved that any non-zero locally nilpotent derivation $D$ on an algebra $A$ with a $\mathbb{Z}$-filtration induces a non-zero locally nilpotent derivation $\operatorname{gr}(D)$ on the graded algebra $\operatorname{Gr}(A)$. In our case, he proved in [8] that $\operatorname{Ker}(\operatorname{gr}(\delta))=\mathbb{C}[x]$.

Recall also that we can define a degree function associated to a locally nilpotent $D \in \operatorname{LND}(A)$ by posing $\operatorname{deg}(0)=-\infty$ and $\operatorname{deg}_{D}(a):=\max \left\{n \in \mathbb{N} \mid D^{n}(a) \neq 0\right\}$ if $a \in A \backslash\{0\}$. Moreover, if $A$ has a $\mathbb{Z}$-filtration, then $\operatorname{deg}_{g r(D)}(g r(a)) \leq \operatorname{deg}_{D}(a)$ for any element $a \in A$. (Here $g r: A \rightarrow \operatorname{Gr}(A)$ denote the natural function from $A$ to $\operatorname{Gr}(A)$.)

This implies that $\operatorname{Ker}(\delta)=\mathbb{C}[x]$. Indeed, if $p \in S_{Q, n} \backslash \mathbb{C}[x]$, then we can choose a filtration on $S_{Q, n}$ such that $\operatorname{gr}(p)$ belongs to $\operatorname{Gr}\left(S_{Q, n}\right) \backslash \mathbb{C}[x]$. Then $\operatorname{Ker}(\delta) \subset \mathbb{C}[x]$ follows from the inequalities $1 \leq \operatorname{deg}_{\operatorname{gr}(\delta)}(\operatorname{gr}(p)) \leq \operatorname{deg}_{\delta}(p)$. Since $\operatorname{Ker}(\delta)$ is of transcendence degree one and is algebraically closed, we obtain that $x \in \operatorname{Ker}(\delta)$.

Thus, $\operatorname{Ker}(\delta)=\mathbb{C}[x]$ for any non-zero locally nilpotent derivation on $S_{Q, n}$. In particular, $\operatorname{ML}\left(X_{Q, n}\right)=\mathbb{C}[x]$. 
Using this result, we can obtain a precise picture of the set of locally nilpotent derivations on rings $S_{Q, n}$ when $n \geq 2$.

Theorem 3.2. Let $X_{Q, n}$ be a Danielewski hypersurface with $n \geq 2$ and let $S_{Q, n}$ denote its coordinate ring. Then

$$
\operatorname{LND}\left(S_{Q, n}\right)=\left\{h(x)\left(x^{n} \frac{\partial}{\partial z}+\frac{\partial Q(x, z)}{\partial z} \frac{\partial}{\partial y}\right), \text { where } h(x) \in \mathbb{C}[x]\right\} .
$$

In particular, $\operatorname{Ker}(\delta)=\mathbb{C}[x]$ and $\operatorname{Ker}\left(\delta^{2}\right)=\mathbb{C}[x] z+\mathbb{C}[x]$ for every non-zero locally nilpotent derivation $\delta \in \operatorname{LND}\left(S_{Q, n}\right)$.

Proof. Let $\delta$ be a non-zero locally nilpotent derivation on $S_{Q, n}$ with $n \geq 2$. In the proof of Theorem 3.1, we showed that $\operatorname{Ker}(\delta)=\mathbb{C}[x]$. Then, due to Lemma 1.1 in [2, there exist polynomials $a(x), b(x) \in \mathbb{C}[x]$ such that $a(x) \delta=b(x) \Delta_{Q, n}$ where $\Delta_{Q, n} \in \operatorname{LND}\left(S_{Q, n}\right)$ is the derivation defined by $\Delta_{Q, n}=x^{n} \frac{\partial}{\partial z}+\frac{\partial Q(x, z)}{\partial z} \frac{\partial}{\partial y}$.

The theorem will follow easily. First note that $a(x) \delta(z)=x^{n} b(x)$. Also $a(x)$ divides $x^{n} b(x)$. Therefore, in order to prove that $a(x)$ divides $b(x)$, it is enough to show that $a(0)=0$ implies $b(0)=0$. This holds since $\delta(y) \in \mathbb{C}[x, z]$ and $a(0) \delta(y)(0, z)=b(0)(Q(0, z))^{\prime}(z)$.

The theorem is proved.

This theorem gives us a very powerful tool for classifying Danielewski hypersurfaces. Indeed, note that an isomorphism $\varphi: A \rightarrow B$, between two algebras $A$ and $B$, conjugates the sets $\operatorname{LND}(A)$ and $\operatorname{LND}(B)$ of locally nilpotent derivations on $A$ and $B$, i.e. $\operatorname{LND}(A)=\varphi^{-1} \operatorname{LND}(B) \varphi$ if $\varphi: A \rightarrow B$ is an isomorphism. In turn, we obtain the following result.

\section{Corollary 3.3.}

(1) Let $\varphi: X_{Q_{1}, n_{1}} \rightarrow X_{Q_{2}, n_{2}}$ be an isomorphism between two Danielewski hypersurfaces with $n_{1}, n_{2} \geq 2$. Then, there exist two constants a, $\alpha \in \mathbb{C}^{*}$ and a polynomial $\beta(x) \in \mathbb{C}[x]$ such that $\varphi^{*}(x)=$ ax and $\varphi^{*}(z)=\alpha z+\beta(x)$.

(2) If $X_{Q_{1}, n_{1}}$ and $X_{Q_{2}, n_{2}}$ are two isomorphic Danielewski hypersurfaces, then $n_{1}=n_{2}$ and $\operatorname{deg}\left(Q_{1}(0, z)\right)=\operatorname{deg}\left(Q_{2}(0, z)\right)$.

(3) Suppose that $X_{Q_{1}, n}$ and $X_{Q_{2}, n}$ are two equivalent Danielewski hypersurfaces with $n \geq 2$, and let $\Phi: \mathbb{C}^{3} \rightarrow \mathbb{C}^{3}$ be an algebraic automorphism such that $\Phi\left(X_{Q_{1}, n}\right)=X_{Q_{2}, n}$. Then, there exist constants $a, \alpha \in \mathbb{C}^{*}, \beta \in \mathbb{C}$ and a polynomial $B \in \mathbb{C}^{[2]}$ such that $\Phi^{*}(x)=$ ax and $\Phi^{*}(z)=\alpha z+\beta+x B\left(x, x^{n} y-Q_{1}(x, z)\right)$.

Proof. For (1) and (2), we follow the ideas of a proof given by Makar-Limanov in [8].

Let $\varphi: X_{Q_{1}, n_{1}} \rightarrow X_{Q_{2}, n_{2}}$ be an isomorphism between two Danielewski hypersurfaces with $n_{1}, n_{2} \geq 2$. Let $x_{i}, y_{i}, z_{i}$ denote the images of $x, y, z$ in the coordinate $\operatorname{ring} S_{i}=S_{Q_{i}, n_{i}}=$ $\mathbb{C}\left[X_{Q_{i}, n_{i}}\right]$.

If $\delta \in \operatorname{LND}\left(S_{1}\right)$, then $\left(\varphi^{*}\right)^{-1} \circ \delta \circ \varphi^{*} \in \operatorname{LND}\left(S_{2}\right)$. Thus, Theorem 3.2 implies $\delta^{2}\left(z_{1}\right)=0$ and $\delta^{2}\left(\varphi^{*}\left(z_{2}\right)\right)=0$ for any locally nilpotent derivation $\delta \in \operatorname{LND}\left(S_{1}\right)$. Therefore, $\varphi^{*}\left(z_{2}\right)=$ $\alpha\left(x_{1}\right) z_{1}+\beta\left(x_{1}\right)$ for some polynomials $\alpha$ and $\beta$. Since $\varphi$ is invertible, $\alpha$ must be a nonzero constant $\alpha \in \mathbb{C}^{*}$.

On the other hand, $\varphi^{*}$ induces an isomorphism $\operatorname{ML}\left(S_{2}\right)=\mathbb{C}\left[x_{2}\right] \rightarrow \operatorname{ML}\left(S_{1}\right)=\mathbb{C}\left[x_{1}\right]$. Consequently, $\varphi^{*}\left(x_{2}\right)=a x_{1}+b$ for some constants $a \in \mathbb{C}^{*}$ and $b \in \mathbb{C}$.

In order to prove $b=0$, consider the locally nilpotent derivation $\delta_{0} \in \operatorname{LND}\left(S_{2}\right)$ defined by $\delta_{0}=\left(\varphi^{*}\right)^{-1} \circ\left(x_{1}^{n} \frac{\partial}{\partial z_{1}}+\frac{\partial Q_{1}\left(x_{1}, z_{1}\right)}{\partial z_{1}} \frac{\partial}{\partial y_{1}}\right) \circ \varphi^{*}$. Now, Theorem 3.2 implies that $\delta_{0}\left(z_{2}\right)$ is divisible by $x_{2}^{n_{2}}$. Since $\delta_{0}\left(z_{2}\right)=a \alpha^{-1}\left(x_{2}-b\right)^{n_{1}}$, we must have $b=0$ and $n_{1} \geq n_{2}$. This proves the first part of the corollary.

Moreover, repeating this analysis with $\varphi^{-1}$ instead of $\varphi$, we also obtain $n_{2} \geq n_{1}$ and so $n_{1}=n_{2}=n$.

Since $\varphi: X_{Q_{1}, n} \rightarrow X_{Q_{2}, n}$ is a morphism, we know that $\varphi^{*}\left(x^{n} y-Q_{2}(x, z)\right)$ belongs to the ideal $\left(x^{n} y-Q_{2}(x, z)\right)$. In particular, when $x=0$, it implies that $Q_{2}(0, \alpha z+\beta(0))=\varphi^{*}\left(Q_{2}(0, z)\right) \in$ $\left(Q_{1}(0, z)\right)$. Thus $\operatorname{deg}\left(Q_{2}(0, z)\right) \geq \operatorname{deg}\left(Q_{1}(0, z)\right)$. 
Working with $\varphi^{-1}$, the same analysis allows us to conclude that $\operatorname{deg}\left(Q_{1}(0, z)\right) \geq \operatorname{deg}\left(Q_{2}(0, z)\right)$. Moreover, it implies that $Q_{2}(0, \alpha z+\beta(0))=\mu Q_{1}(0, z)$ for a certain constant $\mu \in \mathbb{C}^{*}$.

Since the case $n_{1}=n_{2}=1$ was already done by Daigle [3], this suffices to prove the second part of the corollary.

It remains to prove the third part.

Let $X_{Q_{1}, n}$ and $X_{Q_{2}, n}$ be two equivalent Danielewski hypersurfaces with $n \geq 2$, and let $\Phi$ be an algebraic automorphism of $\mathbb{C}^{3}$ such that $\Phi\left(X_{Q_{1}, n}\right)=X_{Q_{2}, n}$.

Since the polynomial $x^{n} y-Q_{1}(x, z)$ is irreducible, there exists a nonzero constant $\mu \in \mathbb{C}^{*}$ so that $\Phi^{*}\left(x^{n} y-Q_{2}(x, z)\right)=\mu\left(x^{n} y-Q_{1}(x, z)\right)$.

Thus, $\Phi$ induces an isomorphism $\Phi_{c}$ between the Danielewski hypersurfaces of equation $x^{n} y-Q_{2}(x, z)=\mu c$ and $x^{n} y-Q_{1}(x, z)=c$ for every $c \in \mathbb{C}$.

Since $n \geq 2$, the Makar-Limanov invariant of these hypersurfaces is $\mathbb{C}[x]$. By (1), we obtain now that the image by $\Phi^{*}$ of the ideal $\left(x, x^{n} y-Q_{2}(x, z)-\mu c\right)$ belongs to the ideal $\left(x, x^{n} y-\right.$ $\left.Q_{1}(x, z)-c\right)=\left(x, Q_{1}(0, z)+c\right)$ for each $c \in \mathbb{C}$. It turns out that

$$
\Phi^{*}(x) \in \bigcap_{c \in \mathbb{C}}\left(x, Q_{1}(0, z)+c\right)=(x) .
$$

Since $\Phi$ is invertible, this implies that $\Phi^{*}(x)=a x$ for a certain constant $a \in \mathbb{C}^{*}$. Thus

$$
-\mu Q_{1}(0, z) \equiv \mu\left(x^{n} y-Q_{1}(x, z)\right) \equiv \Phi^{*}\left(x^{n} y-Q_{2}(x, z)\right) \equiv-Q_{2}\left(0, \Phi^{*}(z)\right) \bmod (x) .
$$

Since $\operatorname{deg} Q_{1}(0, z)=\operatorname{deg} Q_{2}(0, z)$ (by the second part of the corollary), this implies that $\Phi^{*}(z) \equiv$ $\alpha z+\beta \bmod (x)$ for certain constants $\alpha$ and $\beta$ such that $Q_{2}(0, \alpha z+\beta)=\mu Q_{1}(0, z)$.

Thus, we can write $\Phi^{*}(z)=\alpha z+\beta+x B(x, y, z)$, where $B$ is polynomial of $\mathbb{C}[x, y, z]$.

Now, we use again the first part of the corollary. For every $c \in \mathbb{C}$, there exist a constant $\alpha_{c} \in \mathbb{C}^{*}$ and a polynomial $\beta_{c} \in \mathbb{C}^{[1]}$ such that

$$
\Phi^{*}(z)=\alpha z+\beta+x B(x, y, z) \equiv \alpha_{c} z+\beta_{c}(x) \bmod \left(x^{n} y-Q_{1}(x, z)-c\right) .
$$

Therefore, for every $c \in \mathbb{C}$, we have $\alpha_{c}=\alpha, \beta_{c}(0)=\beta$ and

$$
B(x, y, z) \equiv x^{-1}\left(\beta_{c}(x)-\beta\right) \bmod \left(x^{n} y-Q_{1}(x, z)-c\right) .
$$

In particular $B$ has the following property: For infinitely many constants $c \in \mathbb{C}$, there exist polynomials $r_{c}(x) \in \mathbb{C}[x]$ and $s_{c}(x, y, z) \in \mathbb{C}[x, y, z]$ such that

$$
B(x, y, z)=r_{c}(x)+s_{c}(x, y, z)\left(x^{n} y-Q_{1}(x, z)-c\right) .
$$

We will show that any polynomial with this property must belong to $\mathbb{C}\left[x, x^{n} y-Q_{1}(x, z)\right]$. Remark that it suffices to show that at least one polynomial $s_{c}$ belongs to $\mathbb{C}\left[x, x^{n} y-Q_{1}(x, z)\right]$.

In order to see this, we define a degree function $d$ on $\mathbb{C}[x, y, z]$ by posing, for every $f \in$ $\mathbb{C}[x, y, z], d(f):=\operatorname{deg}(\tilde{f}(y, z))$, with $\tilde{f}(y, z)=f(x, y, z) \in \mathbb{C}[x][y, z]$.

Let $B(x, y, z)=r_{c_{0}}(x)+s_{c_{0}}(x, y, z)\left(x^{n} y-Q_{1}(x, z)-c_{0}\right)$ for one $c_{0} \in \mathbb{C}$. Then, $s_{c_{0}}$ satisfies also the above property and its degree $d\left(s_{0}\right)$ is strictly less than $d(B)$.

Therefore, the desired result can be obtained by decreased induction on the degree $d$.

\section{Equivalence Classes}

In this section, we prove the following result.

Theorem 4.1. Two Danielewski hypersurfaces $X_{Q_{1}, n_{1}}$ and $X_{Q_{2}, n_{2}}$ are equivalent if and only if $n_{1}=n_{2}=n$ and there exist $a, \alpha, \mu \in \mathbb{C}^{*}, \beta \in \mathbb{C}$ and $B \in \mathbb{C}^{[2]}$ such that

$$
Q_{2}\left(a x, \alpha z+\beta+x B\left(x, Q_{1}(x, z)\right)\right) \equiv \mu Q_{1}(x, z) \bmod \left(x^{n}\right) .
$$

Remark 4.2. We will show in the next section (Proposition 5.7) that this theorem implies that every Danielewski hypersurface $X_{Q, n}$ with $n \geq 2$ admits at least two non-equivalent embeddings into $\mathbb{C}^{3}$. 
Before proving Theorem 4.1, let us give another result. Given two Danielewski hypersurfaces, it is not easy to check if the second condition in Theorem 4.1 is fulfilled. Therefore, we also show that any Danielewski hypersurface is equivalent to another one which is unique up to an affine automorphism.

\section{Theorem 4.3.}

(1) Every Danielewski hypersurface is equivalent to a Danielewski hypersurface $X\left(p,\left\{q_{i}\right\}_{i=2 . . \operatorname{deg}(p)}, n\right)$ defined by an equation of the form

$$
x^{n} y-p(z)-x \sum_{i=2}^{\operatorname{deg}(p)} p^{(i)}(z) q_{i}(x, p(z)) \quad \text { with } \quad \operatorname{deg}_{x}\left(q_{i}\right)<n-1 .
$$

Moreover, there is an algorithmic procedure which computes, given a Danielewski hypersurface $X$, a hypersurface $X\left(p,\left\{q_{i}\right\}_{i=2 . . \operatorname{deg}(p)}, n\right)$ which is equivalent to $X$.

(2) Two such Danielewski hypersurfaces $X\left(p_{1},\left\{q_{1, i}\right\}_{i=2 . . \operatorname{deg}\left(p_{1}\right)}, n_{1}\right)$ and $X\left(p_{2},\left\{q_{2, i}\right\}_{i=2 . . \operatorname{deg}\left(p_{2}\right)}, n_{2}\right)$ are equivalent if and only if $n_{1}=n_{2}, \operatorname{deg}\left(p_{1}\right)=\operatorname{deg}\left(p_{2}\right)=d$ and there exist some constants $a, \alpha, \mu \in \mathbb{C}^{*}, \beta \in \mathbb{C}$ such that $p_{1}(\alpha z+\beta)=\mu p_{2}(z)$ and $a \alpha^{-i} q_{2, i}(a x, \mu t)=q_{1, i}(x, t)$ for every $2 \leq i \leq d$.

Remark 4.4. This result generalizes the classification of Danielewski hypersurfaces of the form $x^{2} y-z^{2}-x q(z)$ given by Moser-Jauslin and the author in [9].

Proof of Theorem 4.1. Let $X_{Q_{1}, n_{1}}$ and $X_{Q_{2}, n_{2}}$ be two equivalent Danielewski hypersurfaces. Then, the second part of Corollary 3.3 implies $n_{1}=n_{2}=n$.

If $n=1$, the result is already known. Indeed, by Lemma 2.3, every Danielewski hypersurface $X_{Q, 1}$ with $n=1$ is equivalent to a one of the form $X_{p, 1}$ with $p(x, z)=p(z) \in \mathbb{C}[z]$. Then, Daigle [3] has proven that two such hypersurfaces $X_{p_{1}, 1}$ and $X_{p_{2}, 1}$ are isomorphic if and only if $p_{2}(a z+b)=\mu p_{1}(z)$ for some constants $a, \mu \in \mathbb{C}^{*}$ and $b \in \mathbb{C}$.

Now, assume $n \geq 2$ and let $\Phi$ be an automorphism of $\mathbb{C}^{3}$ such that $\Phi\left(X_{Q_{1}, n}\right)=X_{Q_{2}, n}$. Corollary [3.3, gives us constants $a, \alpha \in \mathbb{C}^{*}, \beta \in \mathbb{C}$ and a polynomial $B \in \mathbb{C}^{[2]}$ such that $\Phi^{*}(x)=$ $a x$ and $\Phi^{*}(z)=\alpha z+\beta+x B\left(x, x^{n} y-Q_{1}(x, z)\right)$. Since the polynomial $x^{n} y-Q_{1}(x, z)$ is irreducible, there exists a nonzero constant $\mu \in \mathbb{C}^{*}$ so that $\Phi^{*}\left(x^{n} y-Q_{2}(x, z)\right)=\mu\left(x^{n} y-Q_{1}(x, z)\right)$. It turns out that $Q_{2}\left(a x, \alpha z+\beta+x B\left(x,-Q_{1}(x, z)\right)\right) \equiv \mu Q_{1}(x, z) \bmod \left(x^{n}\right)$, as desired.

Conversely, let $X_{Q_{1}, n}$ and $X_{Q_{2}, n}$ be two Danielewski hypersurfaces with $Q_{2}(a x, \alpha z+\beta+$ $\left.x B\left(x, Q_{1}(x, z)\right)\right) \equiv \mu Q_{1}(x, z) \bmod \left(x^{n}\right)$ for some $a, \alpha, \mu \in \mathbb{C}^{*}, \beta \in \mathbb{C}$ and $B \in \mathbb{C}^{[2]}$.

We pose

$$
R(x, y, z)=x^{-n}\left(Q_{2}\left(a x, \alpha z+\beta+x B\left(x,-x^{n} y+Q_{1}(x, z)\right)\right)-\mu Q_{1}(x, z)\right) \in \mathbb{C}[x, y, z]
$$

and define an endomorphism of $\mathbb{C}^{3}$ by

$$
\Phi(x, y, z)=\left(a x, a^{-n} \mu y+a^{-n} R(x, y, z), \alpha z+\beta+x B\left(x,-x^{n} y+Q_{1}(x, z)\right)\right) .
$$

Remark that $\Phi^{*}\left(x^{n} y-Q_{2}(x, y)\right)=\mu\left(x^{n} y-Q_{1}(x, y)\right)$. Therefore, the theorem will be proved if we show that $\Phi$ is invertible.

It suffices to prove that $\Phi^{*}$ is surjective, i.e.

$$
\mathbb{C}[x, y, z] \subset \Phi^{*}(\mathbb{C}[x, y, z])=\mathbb{C}\left[\Phi^{*}(x), \Phi^{*}(y), \Phi^{*}(z)\right] .
$$

We know already that $x$ and $P_{1}:=x^{n} y-Q_{1}(x, z)$ are in the image of $\Phi^{*}$.

Then, since $z=\alpha^{-1}\left(\Phi^{*}(z)-\beta-x B\left(x,-P_{1}\right)\right)$, we obtain that $z$ belongs to $\Phi^{*}(\mathbb{C}[x, y, z])$.

It remains to show that $y$ belongs to the image of $\Phi^{*}$. To do this, we first remark that there exist polynomials $f, g \in \mathbb{C}^{[3]}$ such that

$$
R(x, y, z)=x y f\left(x, z, P_{1}\right)+g\left(x, z, P_{1}\right) .
$$

Thus, $y\left(\mu+x f\left(x, z, P_{1}\right)\right)=a^{n} \Phi^{*}(y)-g\left(x, z, P_{1}\right) \in \Phi^{*}(\mathbb{C}[x, y, z])$. Now, choose some polynomials $\tilde{f}$ and $\tilde{g}$ such that

$$
\left(\mu+x f\left(x, z, P_{1}\right)\right) \tilde{f}\left(x, z, P_{1}\right)=1+x^{n} \tilde{g}\left(x, z, P_{1}\right) .
$$


Then, we can write

$$
\begin{aligned}
y\left(\mu+x f\left(x, z, P_{1}\right)\right) \tilde{f}\left(x, z, P_{1}\right) & =y\left(1+x^{n} \tilde{g}\left(x, z, P_{1}\right)\right) \\
& =y+\left(P_{1}-Q_{1}(x, z)\right) \tilde{g}\left(x, z, P_{1}\right) .
\end{aligned}
$$

This implies that $y$ belongs to the image of $\Phi^{*}$ and proves the theorem.

Proof of Theorem 4.3. Let $X_{Q, n}$ be a Danielewski hypersurface. Let $p$ and $q$ denote the polynomials such that $Q(x, z)=p(z)+x q(x, z)$.

We can write $q(x, z)$ in the following form: $q(x, z)=\sum_{i=1}^{\operatorname{deg}(p)} p^{(i)}(z) q_{i}(x, p(z))$ for some polynomials $q_{1}, \ldots, q_{\mathrm{deg}(p)} \in \mathbb{C}^{[2]}$.

By Lemma 2.3, we can assume $\operatorname{deg}_{x}\left(q_{i}(x, p(z))\right)<n-1$ for every index $1 \leq i \leq \operatorname{deg}(p)$.

Now, rewrite

$$
Q(x, z)=p(z)+\sum_{k=1}^{n-1} x^{k} \sum_{i=1}^{\operatorname{deg}(p)} p^{(i)}(z) q_{i, k}(p(z))
$$

for suitable polynomials $q_{i, k} \in \mathbb{C}^{[1]}$. Let $1 \leq k_{0} \leq n-1$ be a fixed integer. Then,

$Q\left(x, z-x^{k_{0}} q_{1, k_{0}}(p(z))\right) \equiv p(z)+\sum_{k=1}^{k_{0}} x^{k} \sum_{i=1}^{\operatorname{deg}(p)} p^{(i)}(z) q_{i, k}(p(z))-x^{k_{0}} q_{1, k_{0}}(p(z)) p^{\prime}(z) \quad \bmod \left(x^{k_{0}+1}\right)$.

Therefore, we obtain, using Theorem 4.1, that $X_{Q, n}$ is equivalent to a hypersurface of equation

$$
x^{n} y=p(z)+\sum_{k=1}^{n-1} x^{k} \sum_{i=1}^{\operatorname{deg}(p)} p^{(i)}(z) \tilde{q}_{i, k}(p(z))
$$

with $\tilde{q}_{1, k_{0}}=0$ and $\tilde{q}_{i, k}=q_{i, k}$ if $(i, k) \in\left[1, k_{0}\right] \times[1, \operatorname{deg}(p)] \backslash\left\{\left(1, k_{0}\right)\right\}$. Then, it is easy to prove by induction on $k_{0}$ that $X_{Q, n}$ is equivalent to a hypersurface of the desired form.

Now, we will prove the second part of the theorem.

Let $X_{j}=X\left(p_{j},\left\{q_{j, i}\right\}_{i=2 . . \operatorname{deg}\left(p_{j}\right)}, n_{j}\right), j=1,2$, and pose $Q_{j}=p_{j}(z)+x \sum_{i=2}^{\operatorname{deg}\left(p_{j}\right)} p_{j}^{(i)}(z) q_{j, i}\left(x, p_{j}(z)\right)$.

If $X_{1}$ and $X_{2}$ are equivalent, then, it follows from Theorem 4.1, that $n_{1}=n_{2}=n$ and that there exist $a, \alpha, \mu \in \mathbb{C}^{*}, \beta \in \mathbb{C}$ and $B \in \mathbb{C}^{[2]}$ such that

$$
Q_{2}\left(a x, \alpha z+\beta+x B\left(x, Q_{1}(x, z)\right)\right) \equiv \mu Q_{1}(x, z) \bmod \left(x^{n}\right) .
$$

This implies $p_{2}(\alpha z+\beta)=\mu p_{1}(z)$. Thus $\operatorname{deg}\left(p_{1}\right)=\operatorname{deg}\left(p_{2}\right)=d$.

First, we prove that $B(x, \cdot) \equiv 0 \bmod \left(x^{n-1}\right)$. In order to do this, suppose that we can write $B(x, t) \equiv b_{k}(t) x^{k}$ for some $0 \leq k \leq n-2$ and $b_{k}(t) \in \mathbb{C}[t] \backslash 0$. Then, we obtain the following equalities modulo $\left(x^{k+2}\right)$.

$$
\begin{aligned}
\mu Q_{1}(x, z) & \equiv Q_{2}\left(a x, \alpha z+\beta+x B\left(x, Q_{1}(x, z)\right)\right) \bmod \left(x^{k+2}\right) \\
& \equiv Q_{2}\left(a x, \alpha z+\beta+x^{k+1} b_{k}\left(p_{1}(z)\right)\right) \\
& \equiv p_{2}\left(a x, \alpha z+\beta+x^{k+1} b_{k}\left(p_{1}(z)\right)\right)+a x \sum_{i=2}^{d} p_{2}^{(i)}(\alpha z+\beta) q_{2, i}\left(a x, p_{2}(\alpha z+\beta)\right) \\
& \equiv \mu p_{1}(z)+x^{k+1} b_{k}\left(p_{1}(z)\right)\left(p_{2}\right)^{\prime}(\alpha z+\beta)+a x \sum_{i=2}^{d} p_{2}^{(i)}(\alpha z+\beta) q_{2, i}\left(a x, \mu p_{1}(z)\right) \\
& \equiv \mu p_{1}(z)+x^{k+1} b_{k}\left(p_{1}(z)\right) \alpha^{-1} \mu p_{1}^{\prime}(z)+a x \sum_{i=2}^{d} \alpha^{-i} \mu p_{1}^{(i)}(z) q_{2, i}\left(a x, \mu p_{1}(z)\right) .
\end{aligned}
$$

This would imply

$$
\alpha^{-1}\left(p_{1}\right)^{\prime}(z) b_{k}\left(p_{1}(z)\right) \equiv \frac{\sum_{i=2}^{d}\left(p_{1}^{(i)}(z) q_{1, i}\left(x, p_{1}(z)\right)-a \alpha^{-i} p_{1}^{(i)}(z) q_{2, i}\left(a x, \mu p_{1}(z)\right)\right)}{x^{k}} \bmod \left(x^{k+1}\right),
$$


what is impossible.

Therefore, $B(x, \cdot) \equiv 0 \bmod \left(x^{n-1}\right)$. Since, by hypothesis, $\operatorname{deg}_{x}\left(Q_{j}(x, z)\right)<n$ for $j=1,2$, it follows that $Q_{2}(a x, \alpha z+\beta)=\mu Q_{1}(x, z)$. Then, we can easily check that this last equality implies $a \alpha^{-i} q_{2, i}(a x, \mu t)=q_{1, i}(x, t)$ for every $2 \leq i \leq d$, as desired. This concludes the proof.

\section{STANDARD FORMS}

In [5], A. Dubouloz and the author proved that every Danielewski hypersurface $X_{Q, n}$ where $Q(x, z)$ is a polynomial such that $Q(0, z)$ has $d \geq 2$ simple roots, is isomorphic to a hypersurface of a certain type (called standard form) and then classified all these standard forms up to isomorphism.

In this section, we will generalize these results even when $Q(0, z)$ has multiple roots. In order to do this, we first generalize the definition of standard form given in [5].

Definition 5.1. We say that a Danielewski hypersurface $X_{Q, n}$ is in standard form if the polynomial $Q$ can be written as follows:

$$
Q(x, z)=p(z)+x q(x, z), \quad \text { with } \operatorname{deg}_{z}(q(x, z))<\operatorname{deg}(p) .
$$

We also introduce a notion of reduced standard form.

Definition 5.2. A Danielewski hypersurface $X_{Q, n}$ is in reduced standard form if $\operatorname{deg}_{x}(Q(x, z))<$ $n$ and

$$
Q(x, z)=p(z)+x q(x, z), \quad \text { with } \operatorname{deg}_{z}(q(x, z))<\operatorname{deg}(p)-1 .
$$

When $X_{1}$ and $X_{2}$ are two isomorphic Danielewski hypersurfaces with $X_{2}$ in (reduced) standard form, we say that $X_{2}$ is a (reduced) standard form of $X_{1}$.

Example 5.3.

(1) Danielewski hypersurfaces defined by equations of the form $x^{n} y-p(z)=0$ are in reduced standard form (These hypersurfaces were studied by Makar-Limanov in [8]);

(2) The Danielewski hypersurfaces, studied by Danielewski [4] and Wilkens [10], defined by $x^{2} y-z^{2}-h(x) z=0$ are in standard form;

(3) Danielewski hypersurfaces $X_{\sigma, n}$ defined by equations $x^{n} y=\prod_{i=1}^{d}\left(z-\sigma_{i}(x)\right)$, where $\sigma=\left\{\sigma_{i}(x)\right\}_{i=1 \cdots d}$ is a collection of $d \geq 2$ polynomials, are in standard form; (They are those we have called in standard form in [5])

(4) If $r(x) \in \mathbb{C}[x]$ is a non constant polynomial, then a Danielewski hypersurface defined by $x^{n} y-r(x) p(z)=0$ is not in standard form. (They were studied by Freudenburg and Moser-Jauslin [7].)

We will now prove that every Danielewski hypersurface is isomorphic to a one in reduced standard form. Our proof will be based on the following lemma which comes from [7].

Remark 5.4. Since every Danielewski hypersurface is isomorphic to a one in reduced standard form, the notion of reduced standard form is, in some sense, more relevant than the notion of standard form if we are interested in the classification of Danielewski hypersurfaces. Nevertheless, the notion of standard form has an interest too. Indeed, nice properties are true for every Danielewski hypersurfaces in (not necessarily reduced) standard forms. For example, we will see that all their automorphisms extend to automorphisms of the ambient space. Recall that this does not hold for all Danielewski hypersurfaces. (see [5])

Lemma 5.5. Let $n \geq 1$ be a natural number and $Q_{1}(x, z)$ and $Q_{2}(x, z)$ be two polynomials of $\mathbb{C}[x, z]$ such that

$$
Q_{2}(x, z)=(1+x \pi(x, z)) Q_{1}(x, z)+x^{n} R(x, z)
$$

for some polynomials $\pi(x, z), R(x, z) \in \mathbb{C}[x, z]$.

Then, the endomorphism of $\mathbb{C}^{3}$ defined by

$$
\Phi(x, y, z)=(x,(1+x \pi(x, z)) y+R(x, z), z)
$$

induces an isomorphism $\varphi: X_{Q_{1}, n} \rightarrow X_{Q_{2}, n}$. 
Proof. Remark that, since $\Phi^{*}\left(x^{n} y-Q_{2}(x, z)\right)=(1+x \pi(x, z))\left(x^{n} y-Q_{1}(x, z)\right)$, $\Phi$ induces a morphism $\varphi: X_{Q_{1}, n} \rightarrow X_{Q_{2}, n}$.

Let $f(x, z)$ and $g(x, z)$ be two polynomials in $\mathbb{C}[x, z]$ so that $(1+x \pi(x, z)) f(x, z)+x^{n} g(x, z)=$ 1 and define $\Psi$, an endomorphism of $\mathbb{C}^{3}$, by posing

$$
\left\{\begin{array}{l}
\Psi^{*}(x)=x \\
\Psi^{*}(y)=f(x, z) y+g(x, z) Q_{1}(x, z)-f(x, z) R(x, z) \\
\Psi^{*}(z)=z
\end{array}\right.
$$

We check easily that

$$
\Psi^{*}\left(x^{n} y-Q_{1}(x, z)\right)=f(x, z)\left(x^{n} y-Q_{2}(x, z)\right)
$$

and that

$$
\begin{aligned}
& \Psi^{*} \circ \Phi^{*}(x)=x ; \\
& \Psi^{*} \circ \Phi^{*}(y)=y-g(x, y)\left(x^{n} y-Q_{2}(x, z)\right) ; \\
& \Psi^{*} \circ \Phi^{*}(z)=z .
\end{aligned}
$$

Therefore, the restriction of $\Psi^{*} \circ \Phi^{*}$ to $S_{Q_{2}, n}=\mathbb{C}\left[X_{Q_{2}, n}\right]$ is identity. Hence, $\Psi$ induces the inverse morphism of $\varphi$, and $X_{Q_{1}, n} \simeq X_{Q_{2}, n}$.

Theorem 5.6. Every Danielewski hypersurface is isomorphic to a Danielewski hypersurface in reduced standard form. Furthermore, there is an algorithmic procedure which computes one of the reduced standard forms of a given Danielewski hypersurface.

Proof. Let $X=X_{Q, n}$ be a Danielewski hypersurface and denote $Q(x, z)=p(z)+x q(x, z)$ with $p(z) \in \mathbb{C}[z]$ and $q(x, z) \in \mathbb{C}[x, z]$.

One can construct, by induction on $m \geq 0$, two polynomials $q_{s, m}(x, z)$ and $\pi_{m}(x, z)$ so that $\operatorname{deg}_{z}\left(q_{s}(x, z)\right)<\operatorname{deg}(p)$ and $Q(x, z) \equiv\left(1+x \pi_{m}(x, z)\right)\left(p(z)+x q_{s, m}(x, z)\right) \bmod \left(x^{m+1}\right)$.

Indeed, this assertion is obvious for $m=0$, whereas, if it is true for a rank $m$, we can write:

$$
\begin{aligned}
p(z)+x q(x, z) & \equiv\left(1+x \pi_{m}(x, z)\right)\left(p(z)+x q_{s, m}(x, z)\right) \quad \bmod \left(x^{m+1}\right) \\
& =\left(1+x \pi_{m}(x, z)\right)\left(p(z)+x q_{s, m}(x, z)\right)+x^{m+1} R_{m+1}(x, z) \\
& \equiv\left(1+x \pi_{m}(x, z)+x^{m} \tilde{\pi}_{m+1}(z)\right)\left(p(z)+x q_{s, m}(x, z)+x^{m} r_{m+1}(z)\right) \quad \bmod \left(x^{m+1}\right) \\
& \equiv\left(1+x \pi_{m+1}(x, z)\right)\left(p(z)+x q_{s, m+1}(x, z)\right) \quad \bmod \left(x^{m+1}\right),
\end{aligned}
$$

where $R_{m+1}(0, z)=p(z) \tilde{\pi}_{m+1}(z)+r_{m+1}(z)$ is the Euclidean division (in $\left.\mathbb{C}[z]\right)$ of $R_{m+1}(0, z)$ by $p$.

Thus, we obtain

$$
p(z)+x q(x, z)=\left(1+x \pi_{n-1}(x, z)\right)\left(p(z)+x q_{s, n-1}(x, z)\right)+x^{n} R_{n}(x, z) .
$$

Lemma 5.5 allows us to conclude that $X$ is isomorphic to the Danielewski hypersurface in standard form $X_{s}$ defined by the equation $x^{n} y-p(z)-x q_{s, n-1}(x, z)=0$.

In order to obtain a reduced standard form, we rewrite

$$
p(z)+x q_{s, n-1}(x, z)=\sum_{i=0}^{d} a_{i} z^{i}+x \sum_{i=0}^{d-1} z^{i} \alpha_{i}(x)
$$

and consider the automorphism of $\mathbb{C}^{3}$ defined by

$$
\Phi:(x, y, z) \mapsto\left(x, y, z-x\left(d a_{d}\right)^{-1} \alpha_{d-1}(x)\right) .
$$

One checks that the polynomial $\Phi^{*}\left(x^{n} y-p(z)-x q_{s, n-1}(x, z)\right)$ satisfies the second condition in the definition of Danielewski hypersurface in reduced standard form. Finally, the first condition can be obtain easily by applying Lemma 5.5.

This proof gives an algorithm for finding a (reduced) standard form of given Danielewski hypersurface. 
It should be noticed that a Danielewski hypersurface is in general not equivalent to its (reduced) standard form given by Theorem 5.6. Morover, one can use this fact to construct non-equivalent embeddings for every Danielewski hypersurface of non-trivial Makar-Limanov invariant.

Proposition 5.7. Every Danielewski hypersurface $X_{Q, n}$ with $n \geq 2$ admits at least two nonequivalent embeddings into $\mathbb{C}^{3}$.

Proof. Since, by Theorem 5.6, every Danielewski hypersurface is isomorphic to a one in standard form, it suffices to show that every Danielewski hypersurface in standard form $X_{Q, n}$ with $n \geq 2$ admits at least two non-equivalent embeddings in $\mathbb{C}^{3}$.

Let $X=X_{Q, n}$ be a Danielewski hypersurface in standard form with $n \geq 2$. Then, due to Lemma 5.5, $X$ is isomorphic to the hypersurface $Y=X_{(1+x) Q(x, z), n}$. Nevertheless, it turns out that $X$ and $Y$ are non-equivalent hypersurfaces of $\mathbb{C}^{3}$. Indeed, if they were, Theorem 4.1 would give us constants $a, \alpha, \mu \in \mathbb{C}^{*}, \beta \in \mathbb{C}$ and a polynomial $B \in \mathbb{C}^{[2]}$ such that

$$
(1-a x) Q(a x, \alpha z+\beta+x B(x, Q(x, z))) \equiv \mu Q(x, z) \bmod \left(x^{n}\right) .
$$
$\left(x^{2}\right)$ :

In turn, if we denote $Q(x, z)=p(z)+x q(x, z)$, it would lead the following equalities modulo

$$
\begin{aligned}
\mu Q(x, z) & \equiv \mu(p(z)+x q(0, z)) \\
& \equiv(1-a x) Q(a x, \alpha z+\beta+x B(0, Q(0, z))) \\
& \equiv(1-a x)(p(\alpha z+\beta+x B(0, p(z)))+x q(0, \alpha z+\beta)) \\
& \equiv p(\alpha z+\beta)+x\left(B(0, p(z)) p^{\prime}(\alpha z+\beta)+q(0, \alpha z+\beta)-a p(\alpha z+\beta)\right) .
\end{aligned}
$$

Thus

$$
B(0, p(z)) p^{\prime}(\alpha z+\beta)+q(0, \alpha z+\beta)-a p(\alpha z+\beta)=\mu q(0, z)
$$

which is impossible since $\operatorname{deg}(q(0, z))<\operatorname{deg}(p)$ by definition of a standard form.

Remark 5.8. This proof is similar to the proof of Freudenburg and Moser-Jauslin in [7] for hypersurfaces of equation $x^{n} y=p(z)$ with $n \geq 2$. In their article, they also have constructed non-equivalent embeddings into $\mathbb{C}^{3}$ for Danielewski hypersurfaces of the form $x y-z^{d}-1=0$ for some $d \in \mathbb{N}$. Nevertheless, we do not know if every Danielewski hypersurface $X_{Q, 1}$ admits non equivalent embeddings into $\mathbb{C}^{3}$. For instance, the following question, which they posed in [7], is still open.

Question 1. Does the hypersurface of equation $x y+z^{2}=0$ admit a unique embedding into $\mathbb{C}^{3}$ ?

Remark also that the two non-equivalent embeddings of a Danielewski hypersurface $X_{Q, n}$ with $n \geq 2$ which we construct in Proposition 5.7 are analytically equivalent. Indeed, it can be easily seen, as in [7] and [5], that a Danielewski hypersurface is analytically equivalent to its standard form given by Theorem 5.6. In turn, we obtain the following result.

Proposition 5.9. If $X_{1}$ and $X_{2}$ are two isomorphic Danielewski hypersurfaces, then there is an analytic automorphism $\Psi$ of $\mathbb{C}^{3}$ such that $\Psi\left(X_{1}\right)=X_{2}$.

Proof. Let $X=X_{Q, n}$ be a Danielewski hypersurface and let $X_{Q_{s}, n}$ be its standard form given by the theorem 5.6. In the proof of this theorem, we have seen that $Q(x, z)=(1+$ $x \pi(x, z)) Q_{s}(x, z)+x^{n} R(x, z)$ for certain polynomials $\pi(x, z), R(x, z) \in \mathbb{C}[x, z]$. Now, consider the following analytic automorphism of $\mathbb{C}^{3}$.

$$
\Psi:(x, y, z) \mapsto\left(x, \exp (x f(x, z)) y-x^{-n}(\exp (x f(x, z))-1-x \pi(x, z)) Q_{s}(x, z)+R(x, z), z\right),
$$

where $f(x, z) \in \mathbb{C}[x, z]$ is a polynomial so that $\exp (x f(x, z)) \equiv 1+x \pi(x, z) \bmod \left(x^{n}\right)$. One checks that $\Psi^{*}\left(x^{n} y-Q(x, z)\right)=x^{n} y-Q_{s}(x, z)$. Thus, $\Psi$ maps $X_{Q, n}$ to its standard form 
$X_{Q_{s}, n}$. Then, the result follows from Proposition 6.2, which will be proved at the end of this paper.

\section{Classification up to isomorphism}

Finally, we give the classification of Danielewski hypersurfaces in standard form. Together with the theorem 5.6, this effectively classifies all the Danielewski hypersurfaces up to isomorphism of algebraic varieties.

\section{Theorem 6.1.}

(1) Two Danielewski hypersurfaces $X_{Q_{1}, n_{1}}$ and $X_{Q_{2}, n_{2}}$ in standard form are isomorphic if and only if the two following conditions are satisfied:

(a) $n_{1}=n_{2}=n$;

(b) $\exists a, \alpha, \mu \in \mathbb{C}^{*} \quad \exists \beta(x) \in \mathbb{C}[x]$ such that $Q_{2}(a x, \alpha z+\beta(x)) \equiv \mu Q_{1}(x, z) \bmod \left(x^{n}\right)$.

(2) Two Danielewski hypersurfaces $X_{Q_{1}, n_{1}}$ and $X_{Q_{2}, n_{2}}$ in reduced standard form are isomorphic if and only if the two following conditions are satisfied:

(a) $n_{1}=n_{2}$;

(b) $\exists a, \alpha, \mu \in \mathbb{C}^{*} \quad \exists \beta \in \mathbb{C}$ such that $Q_{2}(a x, \alpha z+\beta)=\mu Q_{1}(x, z)$.

Proof. Let $X_{1}=X_{Q_{1}, n_{1}}$ and $X_{2}=X_{Q_{2}, n_{2}}$ be two isomorphic Danielewski hypersurfaces in standard form and let $\varphi: X_{1} \rightarrow X_{2}$ be an isomorphism. Then Corollary 3.3 implies that $n_{1}=n_{2}=n$. Since the case $n=1$ was already done by Daigle [3], we can suppose that $n \geq 2$.

Denote by $x_{i}, y_{i}, z_{i}$ the images of $x, y, z$ in the coordinate ring $\mathbb{C}\left[X_{i}\right]$ for $i=1,2$. Then, due to Corollary [3.3, there exist constants $a, \alpha \in \mathbb{C}^{*}$ and a polynomial $\beta(x) \in \mathbb{C}[x]$ such that $\varphi^{*}\left(x_{2}\right)=a x_{1}$ and $\varphi^{*}\left(z_{2}\right)=\alpha z_{1}+\beta\left(x_{1}\right)$.

Moreover, we have proven in the proof of Corollary [3.3, that $Q_{2}\left(0, \alpha z_{2}+\beta(0)\right)=\mu Q_{1}\left(0, z_{1}\right)$ for a certain constant $\mu \in \mathbb{C}^{*}$.

Thus, viewing $\mathbb{C}\left[X_{i}\right]$ as a subalgebra of $\mathbb{C}\left[x_{i}, x_{i}^{-1}, z_{i}\right]$ with $y_{i}=x_{i}^{-n} Q_{i}\left(x_{i}, z_{i}\right)$, we obtain

$$
\varphi^{*}\left(y_{2}\right)=\varphi^{*}\left(x_{2}^{-n} Q_{2}\left(x_{2}, z_{2}\right)\right)=\left(a x_{1}\right)^{-n} Q_{2}\left(a x_{1}, \alpha z_{1}+\beta\left(x_{1}\right)\right)=\mu a^{-n} y_{1}+\left(a x_{1}\right)^{-n} \Delta\left(x_{1}, z_{1}\right),
$$

where $\Delta\left(x_{1}, z_{1}\right)=Q_{2}\left(a x_{1}, \alpha z_{1}+\beta\left(x_{1}\right)\right)-\mu Q_{1}\left(x_{1}, z_{1}\right)$.

Remark that $\operatorname{deg}_{z_{1}} \Delta\left(x_{1}, z_{1}\right)<\operatorname{deg}_{z_{1}} Q_{1}\left(0, z_{1}\right)$ since $X_{1}$ and $X_{2}$ are in standard form.

It turns out that $x_{1}^{-n} \Delta\left(x_{1}, z_{1}\right) \in \mathbb{C}\left[x_{1}, z_{1}\right]$ since any polynomial of $\mathbb{C}\left[X_{1}\right] \subset \mathbb{C}\left[x_{1}^{ \pm}, z_{1}\right]$ with negative degree in $x_{1}$ has obviously a degree in $z_{1}$ at least equal to $\operatorname{deg}_{z_{1}} Q_{1}\left(0, z_{1}\right)$. Thus, $\Delta(x, z) \equiv 0 \bmod \left(x^{n}\right)$ and $X_{1}$ and $X_{2}$ fulfill conditions (1) (a) and (1) (b).

If $X_{1}$ and $X_{2}$ are in reduced standard form, then we see easily that $\Delta(x, z) \equiv 0 \bmod \left(x^{n}\right)$ is possible only if $\beta(x) \equiv \beta(0) \bmod \left(x^{n}\right)$. If so $Q_{2}\left(a x_{1}, \alpha z_{1}+\beta(0)\right)=\mu Q_{1}\left(x_{1}, z_{1}\right)$ and $X_{1}$ and $X_{2}$ fulfill the conditions (1) (a) and (2) (b).

Conversely, suppose that $X_{1}=X_{Q_{1}, n} X_{2}=X_{Q_{2}, n}$ are two Danielewski hypersurfaces which satisfy the conditions (a) and (b) of part (1). Then the following triangular automorphism of $\mathbb{C}^{3}$ induces an isomorphism between $X_{1}$ and $X_{2}$ :

$$
(x, y, z) \mapsto\left(a x, \mu a^{-n} y+(a x)^{-n}\left(Q_{2}(a x, \alpha z+\beta(x))-\mu Q_{1}(x, z)\right), \alpha z+\beta\right) .
$$

As a corollary, we observe that two isomorphic Danielewski hypersurfaces in standard form are equivalent via a triangular automorphism of $\mathbb{C}^{3}$, and that two isomorphic Danielewski hypersurfaces in reduced standard form are equivalent via an affine one. In fact, we have even proven a stronger result in the proof of Theorem 6.1,

Proposition 6.2. Every isomorphism between two isomorphic Danielewski hypersurfaces in standard form can be lifted to a triangular automorphism of $\mathbb{C}^{3}$. 


\section{REFERENCES}

[1] Anthony J. Crachiola. On automorphisms of Danielewski surfaces. J. Algebraic Geom., 15(1):111-132, 2006.

[2] D. Daigle. On some properties of locally nilpotent derivations. J. Pure Appl. Algebra, 114(3):221-230, 1997.

[3] Daniel Daigle. Locally nilpotent derivations and Danielewski surfaces. Osaka J. Math., 41(1):37-80, 2004.

[4] W. Danielewski. On a cancellation problem and automorphism groups of affine algebraic varieties. Preprint, Warsaw, 1989.

[5] Adrien Dubouloz and Pierre-Marie Poloni. On a class of Danielewski surfaces in affine 3-space. J. Algebra, 321(7):1797-1812, 2009.

[6] Karl-Heinz Fieseler. On complex affine surfaces with $\mathbf{C}^{+}$-action. Comment. Math. Helv., 69(1):5-27, 1994.

[7] Gene Freudenburg and Lucy Moser-Jauslin. Embeddings of Danielewski surfaces. Math. Z., 245(4):823-834, 2003.

[8] L. Makar-Limanov. On the group of automorphisms of a surface $x^{n} y=P(z)$. Israel J. Math., 121:113-123, 2001.

[9] Lucy Moser-Jauslin and Pierre-Marie Poloni. Embeddings of a family of Danielewski hypersurfaces and certain $\mathbf{C}^{+}$-actions on $\mathbf{C}^{3}$. Ann. Inst. Fourier (Grenoble), 56(5):1567-1581, 2006.

[10] Jörn Wilkens. On the cancellation problem for surfaces. C. R. Acad. Sci. Paris Sér. I Math., 326(9):11111116, 1998.

Mathematisches Institut Universität Basel, Rheinsprung 21, CH-4051 Basel, Switzerland

E-mail address: pierre-marie.poloni@unibas.ch 